\title{
Parameter Estimation Methods for the FOPDT Model, using the Lambert W Function
}

\author{
Radmila Gerov $^{\mathbf{1}}$, Tatjana V Jovanovic ${ }^{\mathbf{2}}$, Zoran Jovanovic ${ }^{1}$ \\ ${ }^{1}$ University of Nis, Faculty of Electronic Engineering, Aleksandra Medvedeva 14, \\ 18000 Nis, Serbia, gerov@ptt.rs, zoran.jovanovic@elfak.ni.ac.rs \\ ${ }^{2}$ University of Nis, Faculty of Medicine, Dr. Zoran Djindjic Boulevard 81, 18000 \\ Nis, Serbia, tatjana@medfak.ni.ac.rs
}

Abstract: This paper discusses the estimation of the processes, that can be described by the first-order plus dead time, system model. Two methods of parameter estimation are proposed in this paper: the closed-loop under the proportional controller step test and the relay feedback test. The standard symmetrical relay and the preload relay, were used with the Relay feedback. Based on the data, from the recorded response, the parameters of the FOPDT model were estimated, by means of manual calculation using the Lambert $W$ function. The produced results were compared to the results using other methods, in the current literature. Illustrated results were shown through a simulation of several different processes, as well as, with the example of the linearized model, of the pupillary light reflex system.

Keywords: Dead-Time System; FOPDT model; Parameters estimations; Relay feedback test; Closed-loop test; Lambert W function; pupillary light reflex system

\section{Introduction}

The main goal of feedback management is for the managed variable to be equal to or at least to deviate as little as possible from the desired value. To create an appropriate controller, which would satisfy all the requirements, it is necessary to understand the behavior of the controlled object in time, i.e. it is necessary to understand the dynamic characteristics of the controlled process. The majority of methods used for designing a controller demand a mathematical process model given in a certain form [1].

Taking into account that processes have different dynamic behavior, they can be described by means of different mathematical models. Models can be described in various ways: with the set of algebraic equations, regular differential equations, non-linear differential equations, etc. The mathematical model needs to portray 
the process' dynamics for its analysis to be comprehensive and accurate. Adequate process control cannot be based on a poor mathematical model, as this would result in a poor design of different controllers, for example: The Linear Quadratic Regulators (LQR) [2], PI controllers [3], PID controllers [4], etc. Choosing an inadequate controller will not only result in unsatisfactory performance values of the controlling system but there is also a chance that the closed-loop control system will become unstable.

On the other hand, there are many processes that we are not acquainted with well enough, processes or systems that are affected by changes in the environment, as well as processes that show highly nonlinear behavior. Difficulties that occur in modeling and identification for this type of cases can be found for example: in robotics problems of modeling and identification of inertia and motor parameters for the PUMA 560 robot [5], difficulties of friction modelling and identification in precision positioning systems [6], parametric sensitivity in chemical reaction systems lead to large change in the temperature of the system [7]. For this reason, it is illusory to expect that in these practical cases a satisfactory, sufficiently precise model of a process or system can always be obtained.

In the past, the wide classes of nonlinear, adaptive, robust and intelligent controllers were developed for this type of systems. Research in adaptive control began in the middle of the last century [8] and was developed into an excellent control tool for many practical applications, e.g. adaptive fuzzy control was used for an uncertain teleoperation system [9]. The robust control approach has been applied in small turbojet engines [10]. Two sliding mode observers tracking control are used for robotic manipulators with uncertain kinematics and dynamics and unknown torques in [11]. Dynamic analysis and intelligent control (fuzzy logic, neural network, genetic algorithm) techniques for flexible manipulators for the time period 1970-2013 are summarized in [12]. Non-linear model predictive control has been used to avoid thermal runaway in semi-batch reactor [13]. For manipulating the feed rate in fed-batch fermentation processes different control strategies can be applied. In [14] was analyzed adaptive control, model predictive control, artificial neural networks and fuzzy control strategies applied in fed-batch fermentation processes.

Apart from the usage of mathematical models for the description of process behavior in the industry, using mathematical models, processes in other fields can be described as well. For example, the dynamics from the light amount reaching the retina to the pupil diameter is described with first-order plus time delay model in [15], the differential equation of the extended tumor growth model is given in [16], the pupil reflex to light has been considered as servomechanism in [17-19], the major features of cell growth and cell cycle in Saccharomyces cerevisiae is described by a coarse-grain model in [20], etc. 
There exist different identification techniques used for determining the mathematical model of a dynamic system [21]. Apart from the usage of mathematical statistics, to process the collected data of a certain process, there can be found two other methods for the estimation of system model parameters in literature. For example, the neuro-fuzzy state-space model obtained from experimental data acquired from a real robotic arm [22], Kohohen's selforganizing maps, examinations of the classification of droplet epitaxial nanostructures [23], vehicle dynamic functions of the cars are obtained from its technical data [24], etc.

It is well known that for a Linear Time-invariant (LTI) system, that by, using the Laplace transformation, differential equations can be transcribed into algebraic equations, in other words, the dynamic characteristics of a process can be described using the transfer function. Most physical processes exhibit nonlinear behavior, but for great number of them the linear time invariant (LTI) models with time delay provide satisfactory model. Furthermore, a great number of controller configuration techniques is based on the system models whose transfer functions are of a lower order, such as first-order plus dead time (FOPDT) model, unstable first-order plus dead time (unstable FOPDT) model, second-order plus dead time (SOPDT) model, integrator plus dead time (IPDT) model, etc. The identification process techniques used for obtaining the previously mentioned lower-order system can be classified into two categories: the time-domain approach, examples [25]-[28], and the frequency domain approach, examples [29-33].

This paper considers parameter estimation of the FOPDT model. Two methods are suggested for the parameter estimation: The closed-loop under the proportional controller step test and the relay feedback test. The transcendental characteristic system equation in both suggested methods is solved by the usage of the Lambert W function (LWF) [34] [35]. The Lambert W function can be evaluated using the open source software the LambertW_DDE Toolbox [36]. Validation of the received FOPDT system model is done using the mean absolute error (MAE) and the root mean squared error (RMSE) index.

With the closed-loop under the proportional controller step test method, the step change is added to the setpoint. From the recorded response, to apply the suggested method, it is necessary to measure five parameters that are going to be used in the estimation of all the FOPDT model parameters [27] [28]. This method is illustrated in the example of the linearized model of the pupillary light reflex system.

The other suggested method for parameter estimation of the FOPDT model uses the standard symmetrical relay feedback test as well as the preload relay feedback test. In order to use this suggested method, it is necessary to measure the values of the amplitude and the oscillation period of the received response [34].

The results were compared to the results received using other methods from current literature. The quality of the suggested methods of parameter estimation of 
the FOPDT model was compared by considering the original system model response and the received FOPDT models within the time and frequency domain.

This paper consists of several sections. The parameter estimation process of the FOPDT model using the closed-loop under proportional controller step test is given in Section 2. Section 3 portrays the parameter estimation process of the FODT model using the relay feedback test. Section 4 portrays the example of estimation with the linearized model of the pupillary light reflex system, while Section 5 presents the Conclusions and compares the results of the suggested methods, to the methods given in the current literature.

\section{Parameter Estimation of FOPDT Model using Closed-Loop under Proportional Controller Step Test}

\subsection{Theoretical Background}

The transfer function of FOPDT model, where $K$ is the gain, $T$ is the time constant and $L$ is dead time, is:

$$
G(s)=\frac{K}{T s+1} e^{-L s}
$$

The closed-loop transfer function $W(s)$ of the FOPDT model under the proportional $(\mathrm{P})$ controller, is:

$$
W(s)=\frac{y(s)}{r(s)}=\frac{K_{p} G(s)}{1+K_{p} G(s)}
$$

where $y(s)$ is the Laplace transform of the controlled output, $r(s)$ is the Laplace transform of the reference step input amplitude $R$ and $K_{p}$ is the gain of the proportional controller.

Substituting the value $G(s)$ given in (1) into the equation in (2), it can be concluded that the closed-loop transfer function:

$$
W(s)=\frac{K_{p} K}{T s+1+K_{p} K e^{-L s}} e^{-L s}
$$

It is well known that the closed-loop poles present the solution to the characteristic system equation. In this particular case, the characteristic equation of the closedloop system is transcendental: 
$T s+1+K_{p} K e^{-L s}=0$

The characteristic system equation, given in (4), because of its transcendental nature has an infinite number of solutions, therefore $W(s)$ has an infinite number of closed-loop poles, which can be calculated using the Lambert $\mathrm{W}$ function:

$s_{k}=\frac{1}{L} W_{k}\left(-\frac{K_{p} K}{T} L e^{\frac{L}{T}}\right)-\frac{1}{T}$

where the $s_{k}$ stands for $k$ number of the closed-loop pole and $k$ stands for an ordinal number of the Lambert $\mathrm{W}$ function branch.

It has been seen in literature, among which are [35] [37], that we can get the rightmost closed-loop poles if the ordinal number of the Lambert $\mathrm{W}$ function branch is equal to 0 or -1 . Taking into account that rightmost closed-loop poles greatly affect the dynamic characteristics i.e. the system response, for the suggested system parameter estimation method, it is enough just to consider them. The rightmost closed-loop poles $\left(s_{1}\right.$ and $s_{2}$ ), are received from the expression given in (5), for $k=0$ and $k=-1$ :

$$
\begin{aligned}
& s_{1}=\frac{1}{L} W_{0}\left(-\frac{K_{p} K}{T} L e^{\frac{L}{T}}\right)-\frac{1}{T} \\
& s_{2}=\frac{1}{L} W_{-1}\left(-\frac{K_{p} K}{T} L e^{\frac{L}{T}}\right)-\frac{1}{T}
\end{aligned}
$$

On the other hand, the closed-loop transfer function $W(s)$, after the approximation of the dead time from the denominator, can be considered as the second-order plus dead time system model with dynamic numerators as shown in [27]. In that case, closed-loop poles, where $\xi$ is a damping ratio and $\omega_{n}$ is natural frequency, are:

$$
\begin{aligned}
& s_{1}=-\xi \omega_{n}+j \omega_{n} \sqrt{1-\xi^{2}} \\
& s_{2}=-\xi \omega_{n}-j \omega_{n} \sqrt{1-\xi^{2}}
\end{aligned}
$$

From equations (6) and (7) we get:

$$
\begin{aligned}
& -\xi \omega_{n}+j \omega_{n} \sqrt{1-\xi^{2}}=\frac{1}{L} W_{0}\left(-\frac{K_{p} K}{T} L e^{\frac{L}{T}}\right)-\frac{1}{T} \\
& -\xi \omega_{n}-j \omega_{n} \sqrt{1-\xi^{2}}=\frac{1}{L} W_{-1}\left(-\frac{K_{p} K}{T} L e^{\frac{L}{T}}\right)-\frac{1}{T}
\end{aligned}
$$




\subsection{Algorithm for Parameter Estimation of the FOPDT Model}

Step 1: By choosing the appropriate proportionate regulator $K_{p}$ gain, we can ensure that the underdamped closed-loop step response is obtained. A typical response is shown in Figure 1.

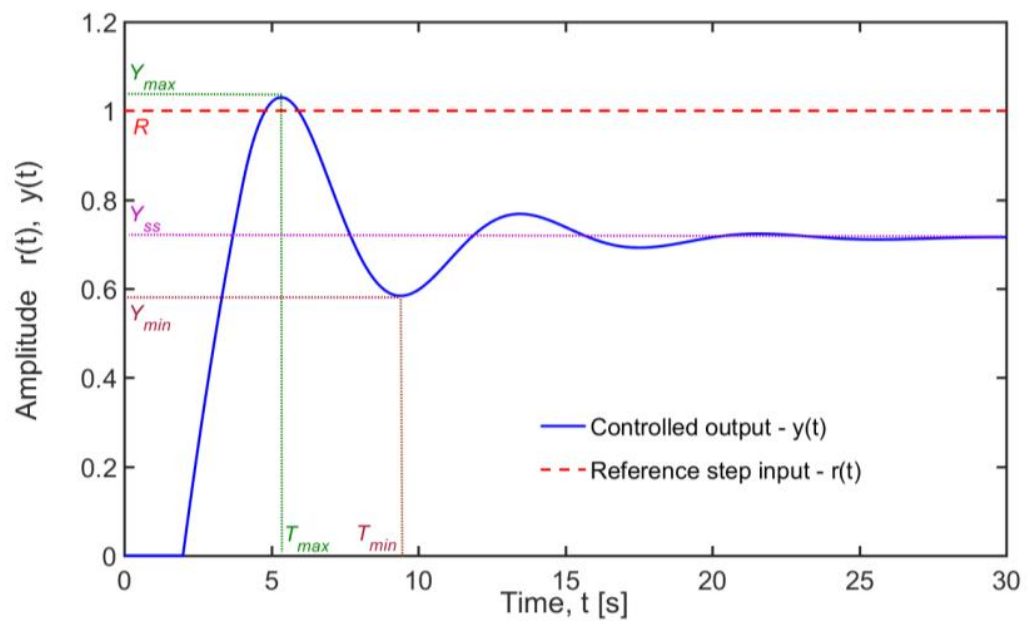

Figure 1

Underdamped closed-loop step response of the FOPDT model under proportional control

Step 2: From the received response, read the values $Y_{\max }, Y_{\min }, Y_{s s}, R, T_{\max }, T_{\min }$, necessary for the application of the suggested estimation [18], as shown in Fig. 1.

Step 3: Calculate the damping ratio $\xi$, natural frequency $\omega_{n}$, and gain $K$ of the FOPDT model using equations (9), (10) and (11), respectively:

$$
\begin{aligned}
& \xi=\frac{-\ln \left(\frac{Y_{s s}-Y_{\min }}{Y_{\max }-Y_{s s}}\right)}{\sqrt{\pi^{2}+\ln \left(\frac{Y_{s s}-Y_{\min }}{Y_{\max }-Y_{s s}}\right)^{2}}} \\
& \omega_{n}=\frac{\pi}{\left(T_{\min }-T_{\max }\right) \sqrt{1-\xi^{2}}} \\
& K=\frac{Y_{s s}}{K_{p}\left(R-Y_{s s}\right)}
\end{aligned}
$$

Step 4: Substitute the received values of damping ratio $\xi$, natural frequency $\omega_{n}$, and gain $K$ of the FOPDT model in the two equations given in (8). Solving the 
system of two equations with two unknowns $T$ - the time constant and $L$-the dead time, all the parameters of the FOPDT model have been estimated.

Step 5: Validate the received FOPDT model using the Mean Absolute Error (MAE) and the Root Mean Squared Error (RMSE) indexes in the time domain, where $y$ is the output of the real system and $y_{m}$ represented output of FOPDT model.

$$
\begin{aligned}
& M A E=\frac{1}{n} \sum_{i=0}^{n}\left|y-y_{m}\right| \\
& R M S E=\sqrt{\frac{1}{n} \sum_{i=0}^{n}\left(y-y_{m}\right)^{2}}
\end{aligned}
$$

Other performance indexes known to literature can also be used for the validation of the received FOPDT model.

\section{Parameter Estimation of the FOPDT Model using the Conventional Symmetrical Relay and the Preload Relay Feedback Test}

\subsection{Theoretical Background}

It is known that the relay belongs to the group of nonlinear elements where static characteristics are described by mathematical models whose complexity depends on the type of nonlinearity. Ambiguous nonlinearities, e.g. relays with hysteresis, are described by functional dependence:

$u(t)=F\left(e(t), \frac{d e(t)}{d t}\right)$

where $u(t)$ is the relay output, $e(t)$ is the output error (the relay input) and $\dot{e}(t)$ is the derivative of $e(t)$. With unambiguous nonlinearities, for example, the ideal relay, $\dot{e}(t)=0$.

Block diagram control system used for the conventional relay feedback test, where $r$ denotes the desired variable (the set-point), $y$ the controlled variable (the process output), $u$ the manipulated variable (the relay output), and $e$ the output error, is shown in Figure 2.

Symmetrical (unbiased) relay and asymmetrical (biased) relay feedback tests are used in the identification process procedure. Techniques based on relay feedback identification methods can be classified into three groups: the describing function 
method, the use of frequency response estimation for model fitting, and the curve fitting approach. The methods used in the paper are the unbiased relay method and the describing function method.

The describing function of the relay represented the complex ratio of the fundamental harmonic relay output to the sinusoidal relay input.

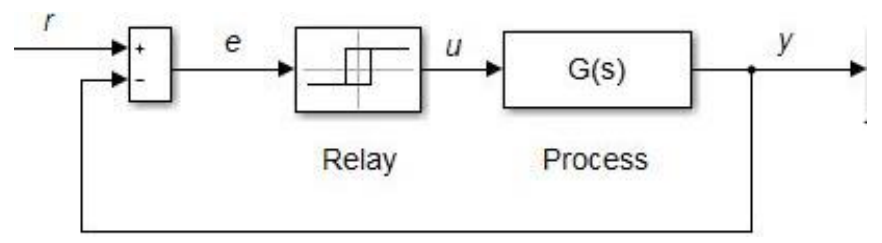

Figure 2

Block diagram of a conventional relay feedback test

The describing function for the unbiased relay with hysteresis $N(A)$ [33], where $h$ is the relay amplitude, $\varepsilon$ is the switch hysteresis and $A$ is the amplitude of the output oscillations, is:

$$
N(A)=\frac{4 h}{\pi A} e^{-j \arcsin \left(\frac{\varepsilon}{A}\right)}=\frac{4 h}{\pi A}\left(\sqrt{\left(1-\frac{\varepsilon^{2}}{A^{2}}\right)}-j \frac{\varepsilon}{A}\right)
$$

The describing function of the relay is a transfer function of a relay for a given frequency. The describing function of the ideal relay is received for the expression in (15) for $\varepsilon=0$. The magnitude of the ideal relay and unbiased relay with hysteresis is equal. As opposed to the ideal relay where the phase is zero, the unbiased relay with hysteresis introduces the phase shift.

It is well known that under an unbiased relay test (the set-point $r(t)=0$ ) the process response moves into the limit cycle. The ultimate gain $K_{u}$ is approximately equal to:

$$
K_{u}=\frac{4 h}{\pi A}
$$

The ultimate frequency $\omega_{u}$, where $P_{u}$ ultimate period, is equal to:

$$
\omega_{u}=\frac{2 \pi}{P_{u}}
$$

The process in Figure 2, is described by the transfer function of the FOPDT model $G(s)$ with the parameters given in (1) and the relay described by the transfer function $N(A)$ given in (15). Characteristic system equation shown in Figure 2 is:

$1+N(A) G(s)=0$ 
Substituting the expression for $G(s)$ given in (1) into equation (18), as well as, using some mathematical arrangement, the following is obtained:

$s_{k}=\frac{1}{L} W_{k}\left(-\frac{N(A) K}{T} L e^{\frac{L}{T}}\right)-\frac{1}{T}$

Taking into account that the process response from Figure 2 moves into the limit cycle under unbiased relay, then there exist two conjugated complex closed-loop poles with real parts equal to zero and imaginary parts $\pm j \omega_{u}$. These poles are rightmost closed-loop poles which can be calculated using the equation (19) for $k=0$ and $k=-1$, therefore:

$$
\begin{aligned}
& +j \omega_{u}=\frac{1}{L} W_{0}\left(-\frac{N(A) K}{T} L e^{\frac{L}{T}}\right)-\frac{1}{T} \\
& -j \omega_{u}=\frac{1}{L} W_{-1}\left(-\frac{N(A) K}{T} L e^{\frac{L}{T}}\right)-\frac{1}{T}
\end{aligned}
$$

If the process response moves into the limit cycle, for ultimate frequency $\omega_{u}$, then:

$$
\left|N(A) G\left(j \omega_{u}\right)\right|=\left|\frac{N(A) K}{\sqrt{\left(\omega_{u} T\right)^{2}+1}}\right|=1
$$

If gain $K>0$. Since the expression under the root in the denominator of the equation (21) is always greater than zero, from (21) it can be seen that:

$$
|N(A)| K=\sqrt{\left(\omega_{u} T\right)^{2}+1}
$$

Substituting the expression (22) into (20), the following is produced:

$$
\begin{aligned}
& +j \omega_{u}=\frac{1}{L} W_{0}\left(-\frac{\sqrt{\left(\omega_{u} T\right)^{2}+1}}{T} e^{-j \arcsin \left(\frac{\varepsilon}{A}\right)} L e^{\frac{L}{T}}\right)-\frac{1}{T} \\
& -j \omega_{u}=\frac{1}{L} W_{-1}\left(-\frac{\sqrt{\left(\omega_{u} T\right)^{2}+1}}{T} e^{-j \arcsin \left(\frac{\varepsilon}{A}\right)} L e^{\frac{L}{T}}\right)-\frac{1}{T}
\end{aligned}
$$

For the ideal relay $\varepsilon=0$, equations (23) are reduced to:

$$
\begin{aligned}
& +j \omega_{u}=\frac{1}{L} W_{0}\left(-\frac{\sqrt{\left(\omega_{u} T\right)^{2}+1}}{T} L e^{\frac{L}{T}}\right)-\frac{1}{T} \\
& -j \omega_{u}=\frac{1}{L} W_{-1}\left(-\frac{\sqrt{\left(\omega_{u} T\right)^{2}+1}}{T} L e^{\frac{L}{T}}\right)-\frac{1}{T}
\end{aligned}
$$


Depending on the type of relay used, the FOPDT model, the time constant $T$, and the dead time $L$ are received by solving the system equations given in (23) or (24). The gain of the FOPDT model, not depending on the type of relay, can be calculated using equation (25):

$$
K=\frac{\pi A}{4 h} \sqrt{\left(\omega_{u} T\right)^{2}+1}
$$

It is well known that by using the unbiased relay feedback test, an error occurs in the estimation of the ultimate gain $K_{u}$, that is, in the magnitude of the describing function of the relay. For this reason, the estimated value of the gain of the FOPDT model, received using equation (25), also deviates from the true value. Value estimation of the gain $K$ is better done using equation (11), for the closedloop under proportional controller step test where $K_{p}<K_{u}$ or using the preload relay [33].

The preload relay can be considered a relay where the proportional controller is connected in parallel to the ideal relay. The gain of the proportional controller is less than the relay amplitude $h$, and is equal $m h$, where $m<0.5$. The describing function of the preload relay is:

$$
N(A)=\frac{4 h}{\pi A}+m h
$$

The gain $K$ of the FOPDT model, using the preload relay feedback test, can be calculated using equation (27) as follows:

$$
K=\frac{\pi A}{h(4+\pi A m)} \sqrt{\left(\omega_{u} T\right)^{2}+1}
$$

In methods known to literature that use the unbiased relay feedback test for the FOPDT model parameter estimation, it is necessary to know either the gain $K$ or the dead time $L$, and the other two parameters are received by solving the two equations. Using the suggested method, it is not necessary to estimate the dead time of the FOPDT model from the limit cycle process response, nor is it necessary to know the gain $K$ of the FOPDT model. It is only necessary to measure the amplitude of the output oscillations $A$ and the ultimate period $P_{u}$.

\subsection{Algorithm for Parameter Estimation of the FOPDT Model using the Relay Feedback Test}

Step 1: Use the unbiased relay feedback test as in Figure 2. The reference input $r(t)$, the ideal relay output $u(t)$, and the process response $y(t)$, is shown in Figure 3 . 
Step 2: From the received response, read the following values: the process response amplitude $A$ and the ultimate period $P_{u}$, for the applied value the relay amplitude $h$, in the manner shown in Figure 3.

Step 3: Calculate the ultimate frequency $\omega_{u}$ using equality in (17).

Step 4: Substitute the received value of the ultimate frequency $\omega_{u}$ into the system of two equations (24). Solving the system equations, the time constant $T$ and the dead time $L$ of the FOPDT model are estimated.

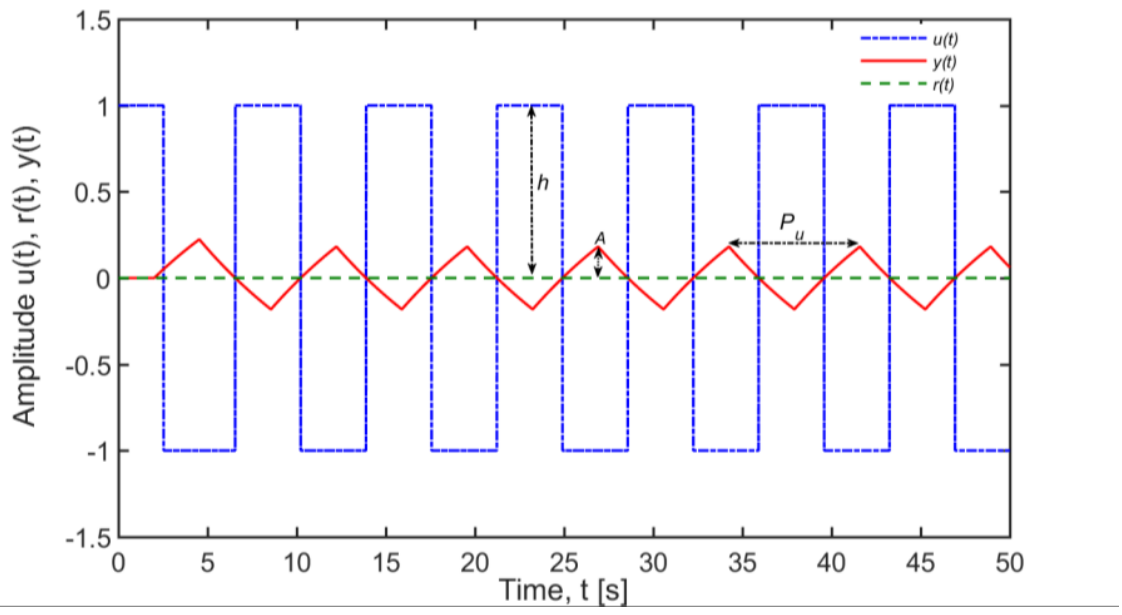

Figure 3

Limit cycle response of FOPDT model under ideal relay feedback test

Step 5: Using equation (25) calculate the gain $K$ of the FOPDT model.

In this way, all parameters of the FOPDT model using the ideal relay feedback test were estimated. With the unbiased relay with the hysteresis feedback test, the only difference is in Step 4 of the suggested algorithm where for the estimation of the time constant $T$ and the dead time $L$ of FOPDT model equations in (23) are used. With the preload relay feedback test in Step 5 of the suggested algorithm, the gain $K$ of the FOPDT model should be calculated using equations in (27).

Step 6: This step can be applied to achieve better estimation results of the gain $\mathrm{K}$ of the FOPDT model. Use the closed-loop under the proportional controller step test for $K_{p}<K_{u}$, as explained in the previous Chapter. From the received response read the steady-state value of the output $Y_{s s}$. For the known value of the amplitude step input $R$, the gain $K$ should be estimated using the equation in (11). 


\section{Simulation Study}

\subsection{Simulation Study using Closed-Loop under Proportional Controller Step Test}

The transfer function of the linearized model of pupillary light reflex [17]-[19], which represents the ratio of the change of flux due to pupil-area alteration Iref $\triangle A$ and change of flux due to external light variation Aref $\Delta I$, is given as the thirdorder plus time delay model:

$$
G(s)=\frac{I_{r e f} \Delta A}{A_{r e f} \Delta I}=\frac{\left(\Delta A / A_{r e f}\right)}{\left(\Delta I / I_{r e f}\right)}=\frac{K e^{-D s}}{(1+\tau s)^{3}}=\frac{0.16 e^{-0.18 s}}{(1+0.1 s)^{3}}
$$

Step 1: Closed-loop unit step response with gain $K_{p}=5$ and the reference step input amplitude $R=1$

Step 2: From the received closed-loop step response the parameters are: $Y_{\max }=0.6330, Y_{\min }=0.36805, Y_{s s}=0.4444, R=1, T_{\max }=0.6735, T_{\min }=1.1165$

Step 3: Calculated parameters from equations (9 thru 11) respectively are: $\xi=0.7892, \omega_{n}=1.7051$, the identified FOPDT model gain $K=0.16$

Step 4: From equation (8) the identified FOPDT model time constant and dead time are: $T=0.2014, L=0.3169$

Step 5: Model validation in time domain (simulation time is 2s): MAE $=0.00308$, RMSE $=0.00513$

The received FOPDT was compared to the FOPDT model received using the Half-rule technique [26] with the following parameters $K=0.16, T=0.15$ and $L=0.33$. Model validation in time domain for Half-rule FOPDT model (simulation time is $2 \mathrm{~s}$ ): $\mathrm{MAE}=0.00240, \mathrm{RMSE}=0.00548$.

Unit step response of the linearized model of pupillary light reflex, proposed identified FOPDT model and Half rule FOPDT model is shown in Figure 4. 


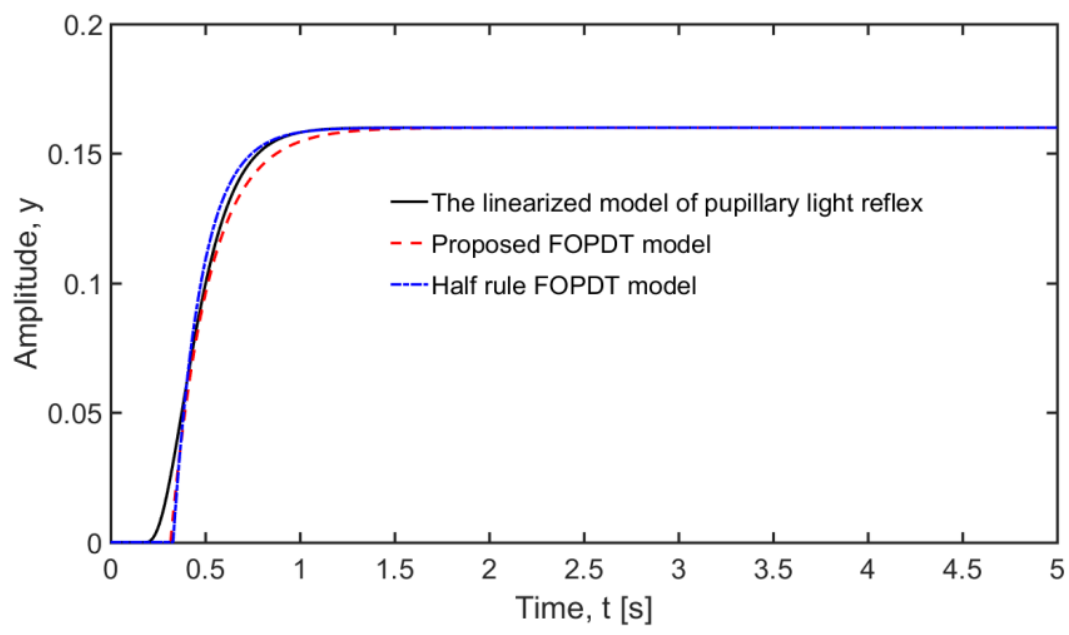

Figure 4

Unit step response of the linearized model of pupillary light reflex and identified FOPDT models

Nyquist fitting of identified FOPDT models for a linearized model of the pupillary light reflex is shown in Figure 5.

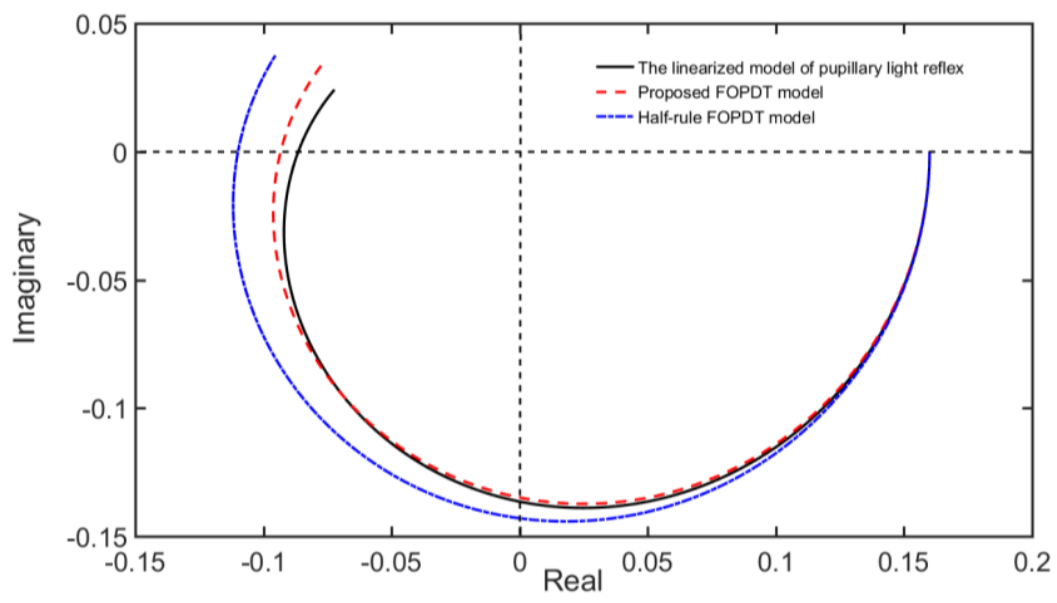

Figure 5

Nyquist fitting of identified FOPDT models for a linearized model of the pupillary light reflex

The gain margin of a linearized model of the pupillary light reflex is $\mathrm{GM}=21.3 \mathrm{~dB}$ and phase crosover freqency $\omega=7.13 \mathrm{rad} / \mathrm{s}$. The same parameteres for the proposed FOPDT model are: $\mathrm{GM}=20.6 \mathrm{~dB}, \omega=6.92 \mathrm{rad} / \mathrm{s}$ and for Half-rule FOPDT models are: $\mathrm{GM}=19.2 \mathrm{~dB}, \omega=7.05 \mathrm{rad} / \mathrm{s}$. 
Based on the given characteristics of the FOPDT model in the time and frequency domain, it can be concluded that the Half-rule FOPDT model represents the characteristics of the linearized model of the pupillary light reflex in time domain more accurately, while the suggested FOPDT model provides better results in the frequency domain.

Comparison results of the suggested parameter estimation method of the FOPDT model with methods from literature are considered in [28] for the following examples: first-order plus time delay system, eight-order plus time delay system and non-minimum-phase time delay system.

\subsection{Simulation Study using Relay Feedback Test}

Parameter estimation of the FOPDT model is given in the example of the WoodBerry distillation column model (WB column) [38] as follows:

$$
G(s)=\frac{12.8}{16.7 s+1} e^{-s}
$$

Step 1: The unbiased relay feedback with the relay amplitude $h=1$

Step 2: From the limited cycle response, the following values were measured: the process response amplitude $A=0.7442$ and the ultimate period $P_{u}=3.9 \mathrm{~s}$.

Step 3: From equations (17) ultimate freqency $\omega_{u}=1.611 \mathrm{rad} / \mathrm{s}$ was calculated.

Step 4: From equation (24) the identified FOPDT model time constant and time delay are: $T=14.005, L=1$.

Step 5: From equation (25) the gain of FOPDT model $K=13.166$.

The identified FOPDT model using the unbiased relay feedback test, is:

$$
G_{1}(s)=\frac{13.166}{14.005 s+1} e^{-s}
$$

Step 6: The closed-loop under proportional controller step test with $K_{p}=1.5$ $\left(K_{p}<1.715\right)$ and the reference step input amplitude $R=1$. From the closed-loop response, the steady state value of the output $Y_{s s}=0.95$. From equation (11) the gain of FOPDT model $K=12.8$.

The identified FOPDT model using the same feedback test and Step 6, is:

$$
G_{2}(s)=\frac{12.8}{14.005 s+1} e^{-s}
$$

Parameter estimation was also performed using the preload relay with describing function (26), with $m=0.2$ and the relay amplitude $h=1$. From the limited cycle response the following values were measured: the process response amplitude 
$A=0.7991$ and the ultimate period $P_{u}=3.89$ s. From equations (17) ultimate freqency $\omega_{u}=1.615 \mathrm{rad} / \mathrm{s}$ was calculated. From equation (24) the identified FOPDT model time constant and time delay are: $T=13.993, L=0.999$. From equation (27) the gain of FOPDT model is $K=12.616$.

The identified FOPDT model using the preload relay feedback test, is:

$$
G_{3}(s)=\frac{12.616}{13.993 s+1} e^{-0.999 s}
$$

Unit step response of the WB column and the identified FOPDT models are shown in Figure 6.

Model validation in time domain (simulation time is $100 \mathrm{~s}$ ): for the identified FOPDT model $G_{l}(s)$ : MAE $=0.65$, RMSE $=0.70$, the identified FOPDT model $G_{2}(s)$ : MAE $=0.34, \mathrm{RMSE}=0.44$, the identified FOPDT model $G_{3}(s): \mathrm{MAE}=0.27$, RMSE $=0.35$.

The gain margin of the $\mathrm{WB}$ column is $\mathrm{GM}=6.44 \mathrm{~dB}$ and ultimate freqency $\omega_{u}=1.61 \mathrm{rad} / \mathrm{s}$. The same parameteres for the FOPDT models $G_{l}(s)$ are: $\mathrm{GM}=4.71$ $\mathrm{dB}, \omega_{u}=1.61 \mathrm{rad} / \mathrm{s}$, for the FOPDT models $G_{2}(\mathrm{~s}), \mathrm{GM}=4.95 \mathrm{~dB}, \omega_{u}=1.61 \mathrm{rad} / \mathrm{s}$ and for the FOPDT models $G_{3}(s), \mathrm{GM}=5.08 \mathrm{~dB}, \omega_{u}=1.62 \mathrm{rad} / \mathrm{s}$.

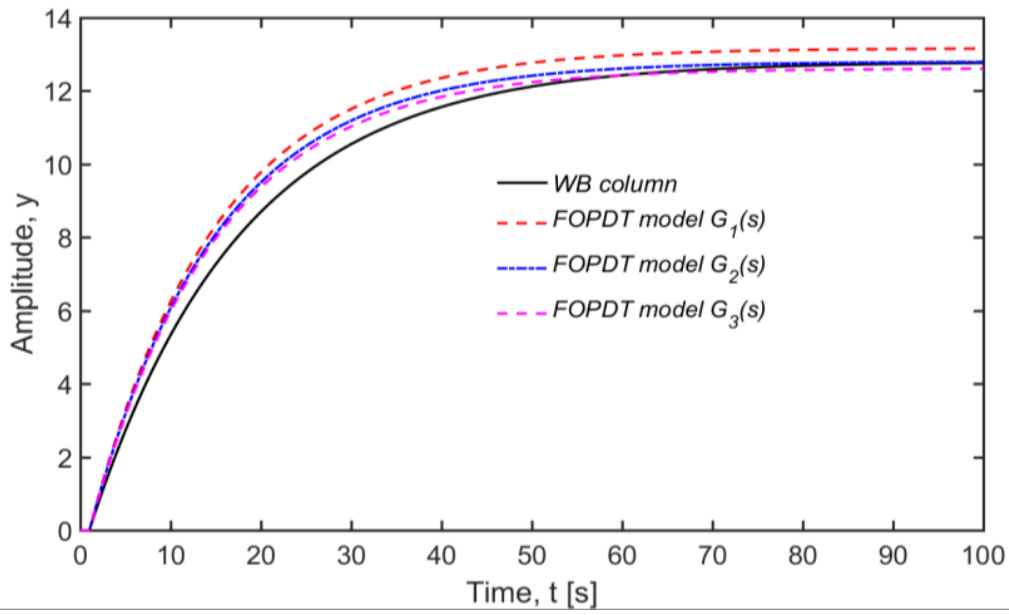

Figure 6

Unit step response of the WB column and identified FOPDT models

The gain margin of the $\mathrm{WB}$ column is $\mathrm{GM}=6.44 \mathrm{~dB}$ and ultimate freqency $\omega_{u}=1.61 \mathrm{rad} / \mathrm{s}$. The same parameteres for the FOPDT models $G_{l}(s)$ are: $\mathrm{GM}=4.71$ $\mathrm{dB}, \omega_{u}=1.61 \mathrm{rad} / \mathrm{s}$, for the FOPDT models $G_{2}(s), \mathrm{GM}=4.95 \mathrm{~dB}, \omega_{u}=1.61 \mathrm{rad} / \mathrm{s}$ and for the FOPDT models $G_{3}(s), \mathrm{GM}=5.08 \mathrm{~dB}, \omega_{u}=1.62 \mathrm{rad} / \mathrm{s}$. 
Nyquist fitting of the identified FOPDT models for a linearized model of WB column is shown in Figure 7.

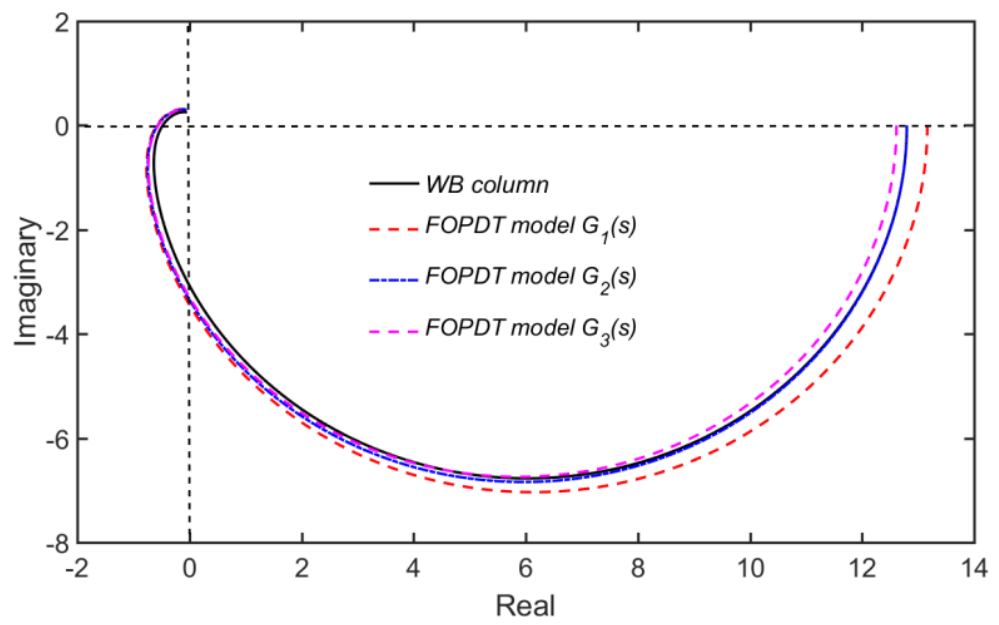

Figure 7

Nyquist fitting of identified FOPDT models for a WB column

It is obvious that the identified FOPDT model $G_{l}(s)$ in comparison to the other two identified models doesn't represent the WB column in time and frequency domain as accurate as the two models. Using Step 6 of the method, the estimated gain is received compared to the gain of the FOPDT model received using formula (25). Using the preload relay feedback test, the parameters of the FOPDT models are better estimated.

\section{Conclusions}

The application of the Lamber $\mathrm{W}$ function, in the unbiased relay test, allows for the estimation of all the parameters of the FOPDT model to be estimated without prior knowledge of either the gain or dead time, as opposed to other methods, in the literature, where it is necessary to know at least one of the two aforementioned parameters, or to read the dead time, from the obtained response.

\section{References}

[1] K. J. Åström, T. Hägglund: PID Controllers: Theory, Design, and Tuning, Research Triangle Park, North Carolina, Instrument Society of America, 1995

[2] R. Zhang, F. Gao, Z. Cao, P. Li: Design and implementation of an improved linear quadratic regulation control for oxygen content in a coke furnace, IET Control Theory and Applications, Vol. 8, No. 14, 2014, pp. $1303-1311$ 
[3] R. Gerov, Z. Jovanovic: Synthesis of PI Controller with a Simple Set-Point Filter for Unstable First-Order Time Delay Processes and Integral plus Time Delay Plant, Elektronika Ir Elektrotechnika, Vol. 24, No. 2, 2018, pp. 3-11

[4] T. Haidegger, L. Kovács, R. E. Precup, B. Benyó, Z. Benyó, S. Preitl: Simulation and control for telerobots in space medicine, Acta Astronautica, Vol. 81, No. 1, 2012, pp. 390-402

[5] P. I. Corke, B. Armstrong-Helouvry: A search for consensus among model parameters reported for the PUMA 560 robot, Proceedings of the 1994 IEEE International Conference on Robotics and Automation, San Diego, CA, USA, Vol. 2, 1994, pp. 1608-1613

[6] L. Márton, B. Lantos: Identification and model-based compensation of Striebeck friction, Acta Polytechnica Hungarica, Vol. 3, No. 3, 2006, pp. $45-58$

[7] J. Jiang, J. Jiang, Z. Wang, Y. Pan: Thermal runaway criterion for chemical reaction systems: A modified divergence method, Journal of Loss Prevention in the Process Industries, Vol. 40, 2016, pp. 199-206

[8] R. Isermann, K. H. Lachmann, D. Matko: Adaptive control systems, Prentice-Hall International, Hemel Hempstead, U.K., 1992

[9] L. Yang, Y. Chen, Z. Liu, K. Chen, Z. Zhang: Adaptive Fuzzy Control for Teleoperation System with Uncertain Kinematics and Dynamics, International Journal of Control, Automation and Systems, Vol. 17, 2019, pp. $1158-1166$

[10] R. Andoga, L. Főző, R. Kovács, K. Beneda, T. Moravec, M. Schreiner: Robust Control of Small Turbojet Engines, Machines, Vol. 7, No. 3, 2019, pp. 1-14

[11] B. Xiao, S. Yin, O. Kaynak: Tracking Control of Robotic Manipulators With Uncertain Kinematics and Dynamics, IEEE Transactions on Industrial Electronics, Vol. 63, No. 10, 2016, pp. 6439-6449

[12] H. N. Rahimi, M. Nazemizadeh: Dynamic analysis and intelligent control techniques for flexible manipulators: a review, Advanced Robotics, Vol. 28, No. 2, 2013, pp. 63-76

[13] Alex Kummer, Tamás Varga, Lajos Nagy: Semi-batch reactor control with NMPC avoiding thermal runaway, Computers \& Chemical Engineering, Vol. 134, 2020, pp. 1-10

[14] L. Mears, S. M. Stocks, G. Sin, K. V. Gernaey: A review of control strategies for manipulating the feed rate in fed-batch fermentation processes, Journal of Biotechnology, Vol. 245, 2017, pp. 34-46

[15] A. Amodio, M. Ermidoro, D. Maggi, S. M. Savaresi: A low-cost system for dynamic analysis of pupillary light response for a driver drowsiness 
detection system, Proceedings IEEE European Control Conference (ECC), 2018, pp. 2691-2696

[16] D. A. Drexler, T. Ferenci, A. Lovrics, L. Kovacs: Tumor dynamics modeling based on formal reaction kinetics, Acta Polytechnica Hungarica, Vol. 16, No. 10, 2019, pp. 31-44

[17] L. Stark: Stability, oscillations, and noise in the human pupil servomechanism, Proceedings of the IRE, Vol. 47, No. 11, 1959, pp. 19251939

[18] L. Stark: Environmental clamping of biological systems: pupil servomechanism, Journal of the Optical Society of America, Vol. 52, No. 8, 1962, pp. 925-930

[19] L. Stark, P. M. Sherman: A servoanalytic study of consensual pupil reflex to light, Journal of Neurophysiology, Vol. 20, 1957, pp. 17-26

[20] P. Palumbo, F. Papa, M. Vanoni, L. Alberghina: A coarse-grain model of growth and cell cycle in Saccharomyces cerevisiae: a mathematical analysis, Acta Polytechnica Hungarica, Vol. 16, No. 10, 2019, pp. 205-224

[21] L. Ljung: System Identification-Theory for the User, Prentice-Hall, Englewood Cliffs, New Jersey, 1987

[22] A. Chatterjee, R. Chatterjee, F. Matsuno, T. Endo: Augmented stable fuzzy control for flexible robotic arm using LMI approach and neuro-fuzzy state space modeling, IEEE Transactions on Industrial Electronics, Vol. 55, No. 3, 2008, pp. 1256-1270

[23] A. Ürmös, Z. Farkas, M. Farkas, T. Sándor, L. T. Kóczy, Á. Nemcsics: Application of self-organizing maps for technological support of droplet epitaxy, Acta Polytechnica Hungarica, Vol. 14, No. 4, 2017, pp. 207-224

[24] A. Szántó, S. Hajdu, G. Á. Sziki: Dynamic Simulation of a Prototype Race Car Driven by Series Wound DC Motor in Matlab-Simulink, Acta Polytechnica Hungarica, Vol. 17, No. 4, 2020, pp. 103-122

[25] Q. Bi, W. J. Cai, E. L. Lee, Q. G. Wang, C. C. Hang, Y. Zhang: Robust identification of first-order plus dead-time model from step response, Control Engineering Practice, Vol. 7, No. 1, 1999, pp. 71-77

[26] S. Skogestad: Simple analytical rules for model reduction and PID controller tuning, Jornal Process Control, Vol. 13, No. 4, 2003, pp. 291-309

[27] R. Gerov, Z. Jovanovic: Parameter Estimation Method for the Unstable Time Delay Process, Acta Polytechnica Hungarica, Vol. 16, No. 3, 2019, pp. 101-120

[28] R. Gerov, Z. Jovanovic: Closed-loop identification of a first-order plus time delay model using Lambert $\mathrm{W}$ function, 2019 IEEE $17^{\text {th }}$ International 
Symposium on Intelligent Systems and Informatics (SISY), Subotica, Serbia, 2019, pp. 000067-000070

[29] Q. Wang, C. Hang, B. Zou, Low-order modeling from relay feedback, Industrial and Engineering Chemistry Research, Vol. 36, No. 2, 1997, pp. $375-381$

[30] W. Li, E. Eskinat, W. Luyben: An improved auto-tune identification method, Industrial and Engineering Chemistry Research, Vol. 30, No. 7, 1991, pp. 1530-1541

[31] T. Liu, F. Gao: Alternative identification algorithms for obtaining a firstorder stable/unstable process model from a single relay feedback test, Industrial and Engineering Chemistry Research, Vol. 47, No. 4, 2008, pp. $1140-1149$

[32] T. Liu, Q. G. Wang, H. P. Huang: A tutorial review on process identification from step or relay feedback test, Journal of Process Control, Vol. 23, No. 10, 2013, pp. 1597-1623

[33] K. Tan, T. Lee, S. Huang, K. Chua, R. Ferdous: Improved critical point estimation using a preload relay, Journal of Process Control, Vol. 16, No. 5, 2006, pp. 445-455

[34] R. Gerov, Z. Jovanović: Parameter Estimation of the IPDT Model using the Lambert W Function, $201914^{\text {th }}$ International Conference on Advanced Technologies, Systems and Services in Telecommunications (TELSIKS), Nis, Serbia, 2019, pp. 400-403

[35] R. Corless, G. Gonnet, D. Hare, D. Jeffrey, D. Knuth: On the Lambert W function, Advances in Computational Mathematics, Vol. 5, 1996, pp. 329359

[36] S. Yi, S. Duan, P. W. Nelson, A. G. Ulsoy: The Lambert W Function Approach to Time Delay Systems and the LambertW_DDE Toolbox, Proceedings of the $10^{\text {th }}$ IFAC Workshop on Time Delay Systems, The International Federation of Automatic Control Northeastern University, Boston, USA, June 22-24, 2012, pp. 114-119

[37] L. Pekař, Q. Gao: Spectrum analysis of LTI continuous-time systems with constant delays: A literature overview of some recent results, IEEE Access, Vol. 6, 2018, pp. 35457-35491

[38] R. Wood, M. Berry: Terminal composition control of a binary distillation column, Chemical Engineering Science, Vol. 28, 1973, pp. 1707-1717 